\title{
An approach for realizing four-wave- mixing experiments stimulated by two-color extreme ultraviolet pulses
}

F. Bencivenga, R. Mincigrucci, F. Capotondi, A. Calvi, R. Cucini, et al.

F. Bencivenga, R. Mincigrucci, F. Capotondi, A. Calvi, R. Cucini, L. Foglia, E. Pedersoli, E. Principi, A. Simoncig, P. Cinquegrana, M. B. Danailov, G. De Ninno, S. Di Mitri, G. Gaio, A. Gessini, L. Giannessi, N. Mahne, M. Manfredda, I. P Nikolov, G. M Penco, L. Raimondi, P. R. Ribič, C. Svetina, M. Trovò, M. Zangrando, C. Masciovecchio, "An approach for realizing four-wave-mixing experiments stimulated by two-color extreme ultraviolet pulses," Proc. SPIE 11886, International Conference on X-Ray Lasers 2020, 118860E (8 July 2021); doi: 10.1117/12.2593345

SPIE. Event: XVII International Conference on X-Ray Lasers, 2020, Online Only 


\title{
An approach for realizing four-wave-mixing experiments stimulated by two-color extreme ultraviolet pulses
}

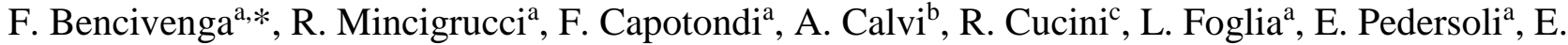

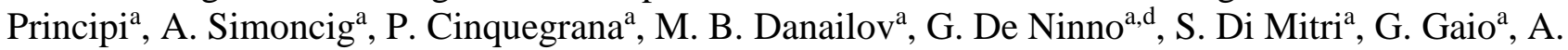 \\ Gessini $^{\mathrm{a}}$, L. Giannessi ${ }^{\mathrm{a}, \mathrm{e}}$, N. Mahne ${ }^{\mathrm{a}, \mathrm{c}}$, M. Manfredda ${ }^{\mathrm{a}}$, I. P. Nikolov ${ }^{\mathrm{a}}$, G. M. Penco ${ }^{\mathrm{a}}$, L. Raimondi ${ }^{\mathrm{a}}$, P. \\ R. Ribič ${ }^{\mathrm{a}}$, C. Svetina ${ }^{\mathrm{f}}$, M. Trovò ${ }^{\mathrm{a}}$, M. Zangrando ${ }^{\mathrm{a}, \mathrm{c}}$, and C. Masciovecchio ${ }^{\mathrm{a}, * *}$

\begin{abstract}
${ }^{a}$ Elettra-Sincrotrone Trieste S.C.p.A., S.S. 14 km 163,5 in Area Science Park, I-34012 Basovizza, Trieste, Italy; ${ }^{b}$ Department of Physics, University of Trieste, Via A.Valerio 2, 34127 Trieste, Italy; ${ }^{c}$ IOM-CNR, S.S. 14 km 163,5 in Area Science Park, I-34012 Basovizza; ${ }^{d}$ Laboratory of Quantum 45, 00044 Frascati, Rome, Italy; ${ }^{\text {P }}$ aul Scherrer Institute, Villigen 5232, Switzerland.
\end{abstract} \\ Optics, University of Nova Gorica, 5001 Nova Gorica, Slovenia; ${ }^{e}$ ENEA CR Frascati, Via E. Fermi
}

*filippo.bencivenga@elettra.eu

**claudio.masciovecchio@elettra.eu

\begin{abstract}
The advent of extreme ultraviolet (EUV) and soft x-ray free electron lasers (FELs) has enabled nonlinear optical experiments at wavelengths shorter than the visible-UV range. An important class of experiments is those based on the four-wave-mixing (FWM) approach, which are often based on interactions between pulses at different wavelengths. The exploitation of multiple EUV/soft x-ray wavelengths is not straightforward, but it can significantly expand the range of applications. In this manuscript we report on an experimental approach, based on the concomitant use of a non-collinear split-delay-and-recombination unit ("mini-Timer") and on a two-color seeded FEL emission scheme ("twin-seed mode"). We used a diamond sample for demonstrating the capability of this setup of generating and detecting a FWM signal stimulated by two-color EUV FEL pulses. This approach can be further exploited for developing experimental methods based on non-linear EUV/x-ray optics.
\end{abstract}

Keywords: non-linear optics, free electron lasers, ultrafast spectroscopy, four wave mixing

\section{INTRODUCTION}

Four-wave-mixing (FWM) processes represent the lowest order non-linear optical response that do not vanish for any sample symmetry and are at the basis of a large array of optical methods and applications. ${ }^{1-5}$ Such experiments rely upon the interactions of three (input) fields driving the $3^{\text {rd }}$ order non-linear polarization to radiate a fourth (output) beam: the FWM signal. The capability to control the parameters of the input fields (frequency, polarization, intensity, time delays, etc.) and to analyze the properties of the output radiation allows probing different features of the sample response, hence leading to a proliferation of methodologies and technological applications.

In the last two decades FWM approaches in the extreme ultraviolet (EUV) and soft x-ray spectral range were thoroughly described on theoretical grounds, showing how major breakthroughs in diverse fields of science could be achieved. ${ }^{5-7}$ Definitely, such a class of experiments might become relevant tools to probe ultrafast dynamics and quantum state correlations inaccessible by linear X-ray methods; their feasibility therefore represents a major challenge for ultrafast X-ray science. ${ }^{8}$ However, the first experimental evidences of non-linear optical responses stimulated by EUV/x-ray pulses ${ }^{9-14}$ have had to await the availability of short-wavelength FEL sources. Indeed, these sources are able to provide ultrafast photon pulses with brightness and coherence comparable with those of optical lasers. In particular, the EUV FWM concept has been applied only recently to develop the FEL-based EUV transient grating (EUV TG) approach, ${ }^{13-20}$ which has been further developed and still represents the unique demonstration of a time-resolved nonlinear optical response driven by EUV or x-ray pulses.

International Conference on X-Ray Lasers 2020, edited by Davide Bleiner, Proc. of SPIE Vol. 11886, 118860E · C) 2021 SPIE · CCC code:

0277-786X/21/\$21 - doi: $10.1117 / 12.2593345$ 
In EUV TG experiments two ultrafast FEL pulses of equal wavelength generate a (transient) sinusoidal spatial modulation of intensity, which launches different kind of dynamics (electronic relaxations, thermal transport, surface and bulk phonons, molecular vibrations, magnetic dynamics, etc.) occurring in a wide range of timescales, from fs to ns, and characterized by a given wavevector imposed by the TG spatial modulation. ${ }^{13-20}$ The interaction between two FEL pulses with different wavelengths, i.e. different photon frequencies $\left(\omega_{1}\right.$ and $\left.\omega_{2}\right)$, can stimulate processes inaccessible by the TG scheme, as for instance coherent Raman scattering, ${ }^{21-24}$ hence substantially broadening the range of potential applications. . $^{-8,14,24}$

In the experiment hereby discussed we employed our non-collinear split-delay-recombination setup ("miniTIMER"), ${ }^{25-27}$ previously used for realizing EUV TG, ${ }^{14-18}$ and the two-color seeded FEL emission ("twin-seed mode" $)^{24,28}$ available at the FERMI FEL1 source, ${ }^{29}$ to demonstrate a time-resolved FWM response driven by the interaction between two EUV pulses at different photon frequencies.

\section{EXPERIMENTAL SETUP}

\subsection{Experimental design}

The concept of the two-color EUV FWM experiment is sketched in Figure 1a, while the pulse sequence is illustrated in Figure $1 \mathrm{~b}$. A sequence of two FEL pulses, separated by a time delay $\Delta \mathrm{t}_{\mathrm{FF}} \approx 300 \mathrm{fs}$, with similar intensity ( $\mathrm{I}_{1}$ $\approx \mathrm{I}_{2}$ ), bandwidth $\delta \omega \approx 30 \mathrm{meV}$ (full-width-at-half-maximum, FWHM) and estimated pulse duration, $\delta \mathrm{t}_{\mathrm{F}}$, in the range 50$70 \mathrm{fs}$, interact with a diamond crystal. The photon energy of the first pulse was $\omega_{1}=47.51 \mathrm{eV}$, while that of the second pulse was $\omega_{2}=\omega_{1}+\Delta \omega$, where $\Delta \omega \approx 270 \mathrm{meV}$. A typical spectrum of the FEL pulses, highlighting the high spectral purity of the "twin-seed" two-color FEL emission, is shown in the inset of Figure 1b. We then used our special splitdelay-recombination setup ("mini-Timer"), ${ }^{25-27}$ which is available at the DiProI beamline, ${ }^{30}$ to: (i) generate two spatially separated FEL beams, hereafter labeled with the superscripts A and B, (ii) overlap the two beams on the diamond sample with a crossing angle $2 \theta \approx 6$ degree and (iii) introduce a time-delay equal to $\Delta \mathrm{t}^{\mathrm{FF}}$ in one of the two sequences in order to superimpose in time the first pulse $\left(\omega_{1}{ }^{\mathrm{A}}\right)$ of one sequence with the second pulse $\left(\omega_{2}{ }^{\mathrm{B}}\right)$ of the other sequence. The choice of a diamond sample was mainly motivated by the high value of the $\chi^{(3)}$ (in the optical range), by the availability of EUV TG data, ${ }^{17}$ the transparency to the optical probe, the easiness of handling and the robustness to radiation damage. The FEL beams were focused at the sample on $\mathrm{a} \approx 0.02 \mathrm{~mm}^{2}$ spot size. The intensity of the FEL pulses $\left(\mathrm{I}_{1,2}{ }^{\mathrm{A}, \mathrm{B}}\right)$ at the sample position was varied in the $0.7-2.0 \mu \mathrm{J}$ range. The third input field needed to stimulate the FWM response was emitted by an optical laser $\left(\omega_{\text {opt }}=3.16 \mathrm{eV}\right.$, time duration $\approx 100 \mathrm{fs}$, pulse energy $\mathrm{I}_{\text {opt }} \approx 1 \mu \mathrm{J}$, spot size $\left.\approx 0.005 \mathrm{~mm}^{2}\right)$, coplanar to the two FEL beams and impinging onto the sample at an incidence angle $\theta_{\text {opt }} \approx 45^{\circ}$. All input fields were polarized orthogonally to the scattering plane. The FEL repetition rate was $10 \mathrm{~Hz}$. Note that the presence of the two additional FEL pulses, $\omega_{2}{ }^{\mathrm{A}}$ and $\omega_{1}{ }^{\mathrm{B}}$ (see Figure $1 \mathrm{~b}$ ), that are not directly involved in the FWM process is unavoidable. A CCD detector (MTE-2048, Princeton Instruments) was placed along the $\mathrm{k}_{\text {out }}=\mathrm{k}_{1}{ }^{\mathrm{A}}-\mathrm{k}_{2}{ }^{\mathrm{B}}+\mathrm{k}_{\mathrm{opt}}$ "phase matched" direction, where we expected to observe the FWM signal stimulated by the interaction between the two FEL pulses and the optical one; a $2 \mathrm{x} 2$ binning of the pixels was used.

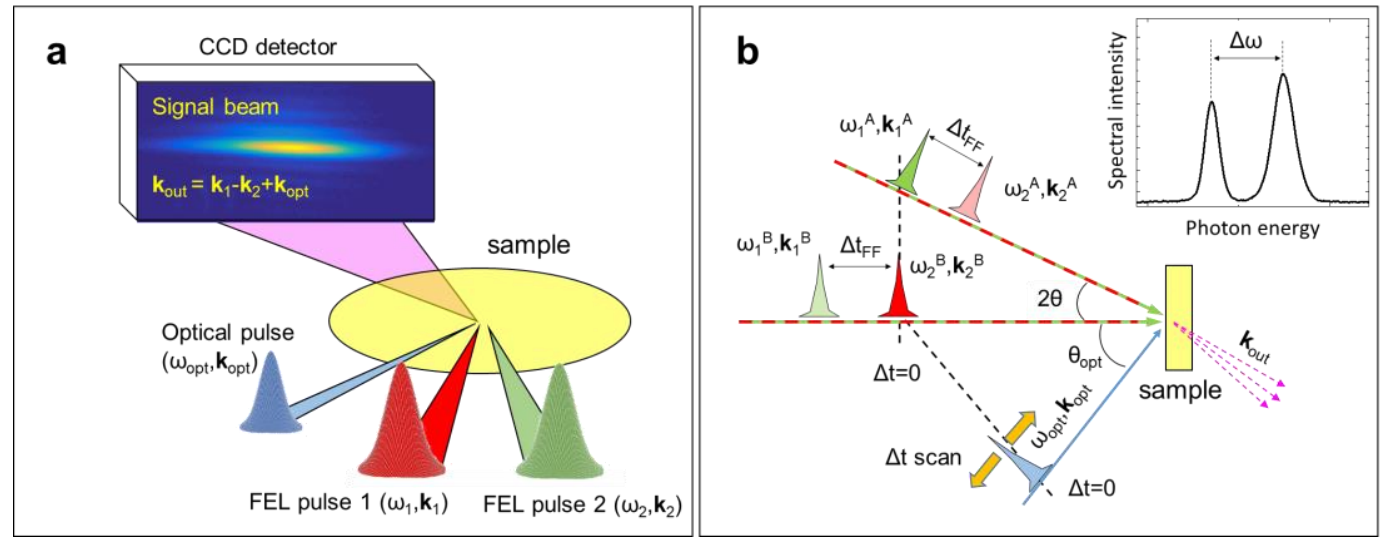

Figure 1: (a) Sketch of the two-color EUV FWM experiment. (b) Employed pulse sequence; see the text for the notation. The inset shows the typical spectrum of two-color FEL emission. 


\subsection{Two-color FEL emission}

Figure 2 sketches the employed experimental setup. The electron beam emerging from the linear accelerator (LINAC) was seeded by a sequence of two seed pulses, derived by the same IR laser pulse $\left(\omega_{\mathrm{IR}} \approx 1.58 \mathrm{eV}\right)$. One of these pulses is frequency up-converted by a third harmonic generation (THG) stage, resulting in $\omega_{\text {seed } 2} \approx 4.75 \mathrm{eV}$, while the other is used to pump an optical parametric amplifier (OPA), which permits to vary the photon frequency of this seed in a range $\omega_{\text {seed } 1} \approx 4.75-5.17 \mathrm{eV}$. These two seeding pulses are then time-delayed (by $\Delta \mathrm{t}_{\mathrm{FF}}$ ), collinearly coupled and focused into the modulator, where they interact with an electron bunch with flat phase-space. The temporal length of the electron bunch was about $0.7 \mathrm{ps}$ in order to accommodate both seeds. The temporal and spectral separation between the two seed pulses can be controlled, respectively, by acting on a delay stage (DS) and on the OPA settings, and can be monitored by cross-correlation and an optical spectrometer (CC+OS); the pulse duration of the THG seeding pulse was slightly shorter than the OPA one ( $\approx 100 \mathrm{fs}$ vs $\approx 150 \mathrm{fs}$ FWHM), due to a residual chirp in the OPA pulse. It was possible to mechanically shutter the two pulses independently and, furthermore, it is worth mentioning the possibility to keep constant $\Delta t_{\mathrm{FF}}$ by acting on the DS. The two portions of the electron bunch that have interacted with the seed pulses emit FEL radiations at the $\mathrm{N}^{\text {th }}$ harmonic when run through the radiators $;{ }^{28}$ in the present experiment the radiators were tuned at harmonic 10. The final output hence consists into two distinct FEL pulses, generated by the two distinct seeds and separated in time by $\Delta t_{\mathrm{FF}}$. The main limitations of such a "twin-seed" scheme are the spectral $(\Delta \omega)$ and temporal $\left(\Delta \mathrm{t}_{\mathrm{FF}}\right)$ separation in between the two final FEL pulses. The former cannot exceed the gain bandwidth of the FEL $\left((\Delta \omega / \omega)_{\text {FEL }} \approx\right.$ $0.5 \%$ ), which in the present case results into $\Delta \omega<0.3 \mathrm{eV}$, while $\Delta \mathrm{t}_{\mathrm{FF}}$ cannot be longer than the dime duration of the electron bunch $^{31}(\approx 0.7 \mathrm{ps})$ nor shorter than the time duration of the seed pulses. Indeed, a temporal overlap between the two seeds results into the rapid growth of multiple lines in the FEL spectrum and in a deterioration of the stability of the FEL emission.

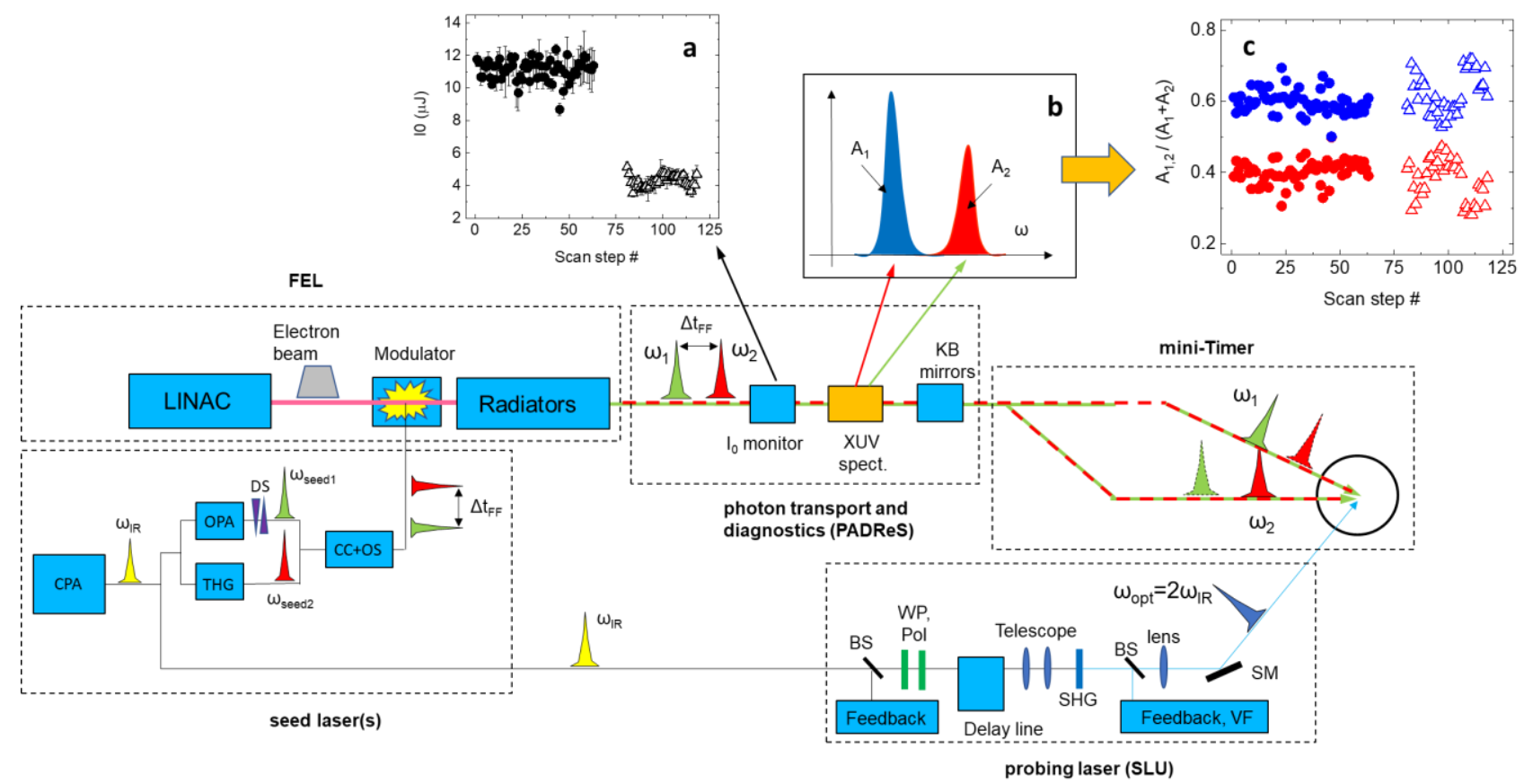

Figure 2: The experimental setup is sketched in the main panel, dashed rectangles enclose the principal elements, i.e.: the seed laser(s), the FEL, the photon transport and diagnostics (PADReS), the mini-Timer setup and the probing laser (SLU). Sub-panels show data from the main online FEL diagnostics, namely: the I0 monitor (panel a), which measures the total FEL intensity, and the EUV energy spectrometer (panel b). Once known the total FEL intensity, from the analysis of the FEL spectrum one can determine the relative intensities of the two-color FEL pulses, shown in panel $\mathbf{c}$ as red and blue symbols; circles and triangles in panels a and $\mathbf{c}$ correspond to two different scans, carried out at a different FEL flux levels. 


\subsection{Optical probe}

Before generating the two seed pulses, a fraction of the output of a Ti:sapphire chirped pulsed amplifier (CPA) is transported downstream to the experimental hall and acts as probing laser, termed "seed laser for users" (SLU); see Figure 2. The SLU pulse is inherently synchronized with the FEL, down to a $\approx 10 \mathrm{fs}$ jitter, ${ }^{32}$ while the long-term trajectory and timing drifts due to the long $(\approx 100 \mathrm{~m})$ IR transport system are stabilized by feedback systems. ${ }^{33}$ Since the jitter level is negligible as compared with the time duration of the employed pulses, the use of "timing tools" for sorting the FEL-optical arrival time is not needed. This situation represents a remarkable practical advantage related to the use of a seeded FEL. A set of waveplates and polarizers (WP, Pol), located close to the experimental chamber, are used to control the intensity and polarization of the optical beam, which is then time delayed by a mechanical delay-line and frequency up-converted by a second harmonic generation (SHG) crystal. The beam is focused at the sample position at about $45^{\circ}$ angle of incidence by a lens and a steering mirror (SM); the input trajectory into the end station is stabilized by a feedback locked to a virtual focus (VF).

\subsection{FEL photon beam control}

The FEL output was monitored by the photon transport and diagnostics system (PADReS); see Figure $2 .{ }^{34}$ The FEL intensity and spectral content were measured, shot-by-shot, by a gas-based intensity monitors (IOMs) and by an online energy spectrometer (PRESTO), ${ }^{35}$ respectively. The photon beam, after the diagnostics section, was directed to the end-station through the photon transport system and a Kirkpatrick-Baez active optics system (KAOS) was used to properly adjust the focusing conditions in order to match the spot size to the experimental requests. The combination of IOMs and spectrometer data permitted the determination of the intensity of the (spectrally separated) FEL pulses: $I_{1,2}=I 0 * A_{1,2} /\left(A_{1}+A_{2}\right)$, where $A_{1,2}$ are the areas under the two FEL spectral lines; see Figures 1a-1c. The seeding scheme adopted at FERMI permitted to achieve high stability in the two-color FEL emission, with respect to what achievable in FEL sources not stabilized by the seeding process. Typical fluctuations in the photon parameters of major interest, i.e. $\mathrm{I}_{1} / \mathrm{I}_{2}, \delta \omega$ and $\Delta \omega$, were about $20 \%, 4 \%$ and $3 \%$, respectively.

As mentioned above, the non-collinear recombination of the two-color FEL pulses was made by a special setup, described in details elsewhere ${ }^{7,27,28}$ and sketched in Figure 3a, consisting in three plane EUV mirrors (M0, M1 and M2) able to split the FEL pulse into two halves and recombine them at the sample position with a selected crossing angle (20). The system allows for a tuning of EUV mirror positions and angles. Such movements can be combined in order to control the arrival time of the FEL beam propagating through the "M0-M2-sample" path (variable delay branch, VDB) with respect to the one propagating along the "M0-M1-sample" path. The simultaneous change of the pitch angles $(\alpha)$ of M0 and M2 (by the same amount) accompanied by a translation of M2 along the M2-sample direction permits to apply a delay between the two beam paths while keeping fixed 20; see Figure 3. For the present experiment the stroke of the M2 translation was purposely extended in order to apply a time delay up to $\approx 500 \mathrm{fs}$ in the VDB, hence permitting to compensate for the intrinsic delay $\left(\Delta \mathrm{t}_{\mathrm{FF}} \approx 300 \mathrm{fs}\right.$ ) between the two color FEL pulses. The procedure to change the delay in the VDB was carried out "step-by-step", by tuning the OPA output at the same photon energy as the THG (i.e. $\omega_{\text {seed } 1}=$ $\left.\omega_{\text {seed2}}\right)$ and by monitoring and optimizing the EUV TG signal at each intermediate step; note that since the wavelength of the two FEL pulses was the same, we could not determine the relative intensities of the two pulses. To facilitate the time overlap procedure both the FEL intensity and pulse length were increased, in order to have a residual temporal overlap between the two EUV pulses, which guaranteed a very weak (but still appreciable) EUV TG signal; see Figure 3c. After the time overlap procedure between the "head pulse" from the VDB with the "tail pulse" from the fixed (M0-M1sample) path we decreased the strength of the FEL dispersive section, changed the OPA wavelength (retrieving the information on the intensity of the two pulses) and optimized the alignment of the system by maximizing the EUV FWM signal. 

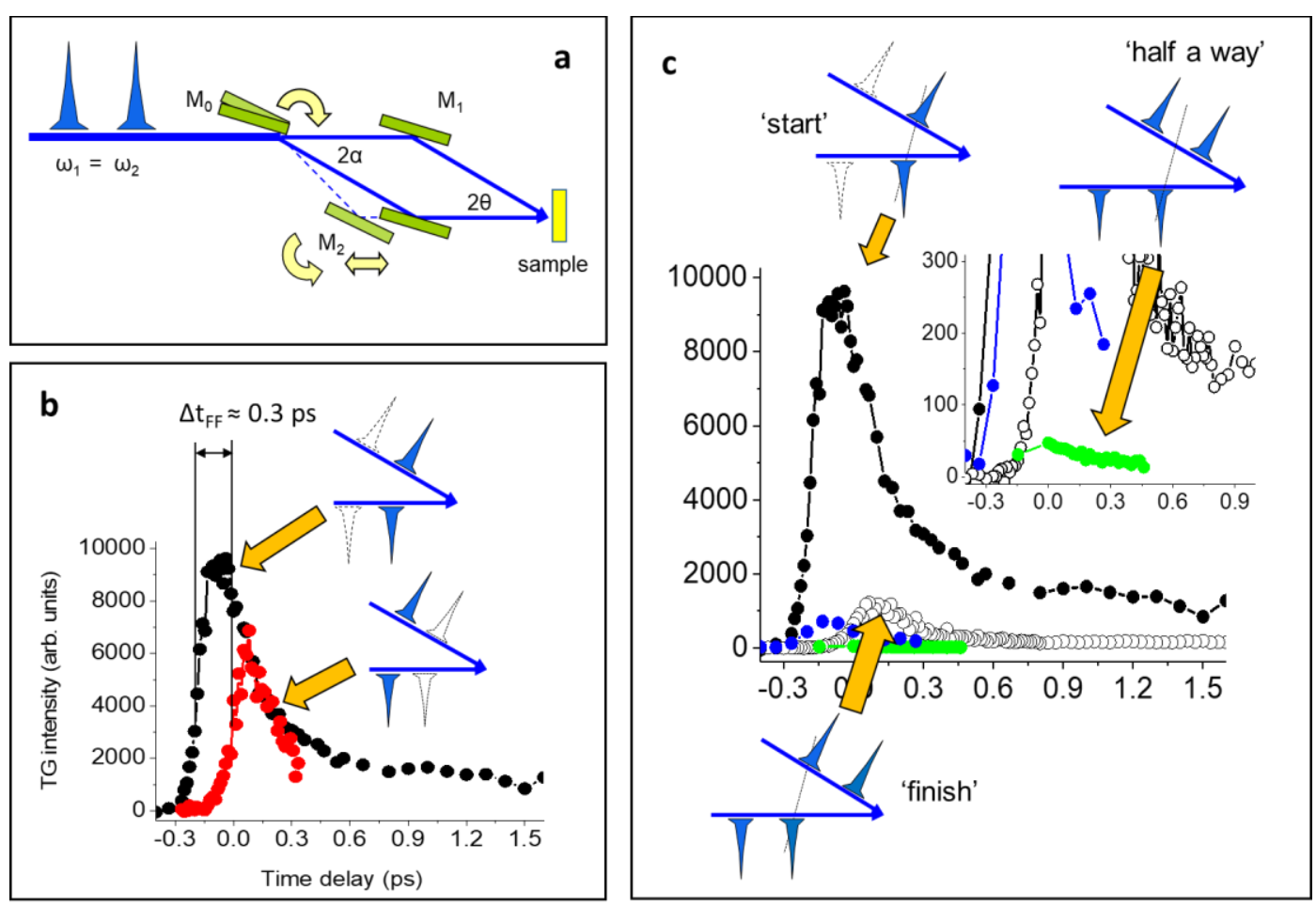

Figure 3: (panel a) sketch of the mini-Timer setup and the combination of movements (M0 pitch / M2 pitch / M2 translation) used to compensate for the time delay between the two FEL pulses radiate in the "twin-seed" mode. (panel b) The black and red circles connected by lines are EUV TG data acquired by using the "head" and "tail" FEL pulses, respectively. The evident shift in the time delay is due to the time-separation $\left(\Delta \mathrm{t}_{\mathrm{FF}}\right)$ between the two FEL pulses. (panel c) Black circles connected by lines are as in panel b, blue circles connected by lines correspond to the first step of the procedure to overlap in time the two-color FEL pulses, green ones roughly correspond to the maximum time separation between the two FEL pulses (the large difference in the EUV TG signal intensity can be better appreciated in the inset), while open black circles connected by lines are the final step. The inclined black-dotted line across the FEL pulse sequences "tracks" the arrival time of the "head" FEL pulse in the VDB.

\section{RESULTS}

When the time delay $(\Delta t)$ of the optical pulse with respect to the time-coincident two-color FEL pulses was set to zero, we observed the footprint of the FWM signal beam in the CCD detector. The signal disappeared when one of the two FEL pulse sequences (i.e. either $\omega_{1}{ }^{\mathrm{A}}$ and $\omega_{1}{ }^{\mathrm{B}}$ or $\omega_{2}{ }^{\mathrm{A}}$ and $\omega_{2}^{\mathrm{B}}$ ) was not present, as shown in Figure 4. Since the two FEL emission lines are spectrally separated and the temporal separation in between the seed pulses prevents spectral contaminations among the two FEL pulses, the experimental observation reported in Figure 4 is sufficient for inferring that this signal arose from the $3^{\text {rd }}$-order non-linear interaction (FWM processes) between the optical field and the twocolor FEL pulses. This undoubtedly demonstrates the feasibility of multi-color FEL-based FWM experiments from solidstate samples and represent the main result of the present work. A preliminary study of the dependence of such a signal beam on $\Delta t, \Delta \omega$ and on the FEL flux was also carried out; it will be part of a subsequent publication. Here we further mention some of the limitations of the present setup, which can be overcome for improving its capabilities in the context of multi-color FWM experiments. One of the main limits during the present experiment was the inability to determine the spectrum of the signal beam, nor to estimate it via the precise determination of the emission angle. Recently we have successfully tested a compact in-vacuum optical spectrometer, ${ }^{36}$ which can be used to spectrally resolve the FWM signal. Another limit was the impossibility to precisely scan the time delay between the two FEL pulses, which represents a relevant variable for FWM experiments and is now implemented as a standard in the mini-Timer setup. On the other hand, an inherent limit of the present approach is the presence of the "additional" FEL pulses, $\omega_{1}{ }^{\mathrm{B}}$ and $\omega_{2}{ }^{\mathrm{A}}$ (see Figure 4), which do not contribute to the FWM signal but cannot be a priori ignored. 

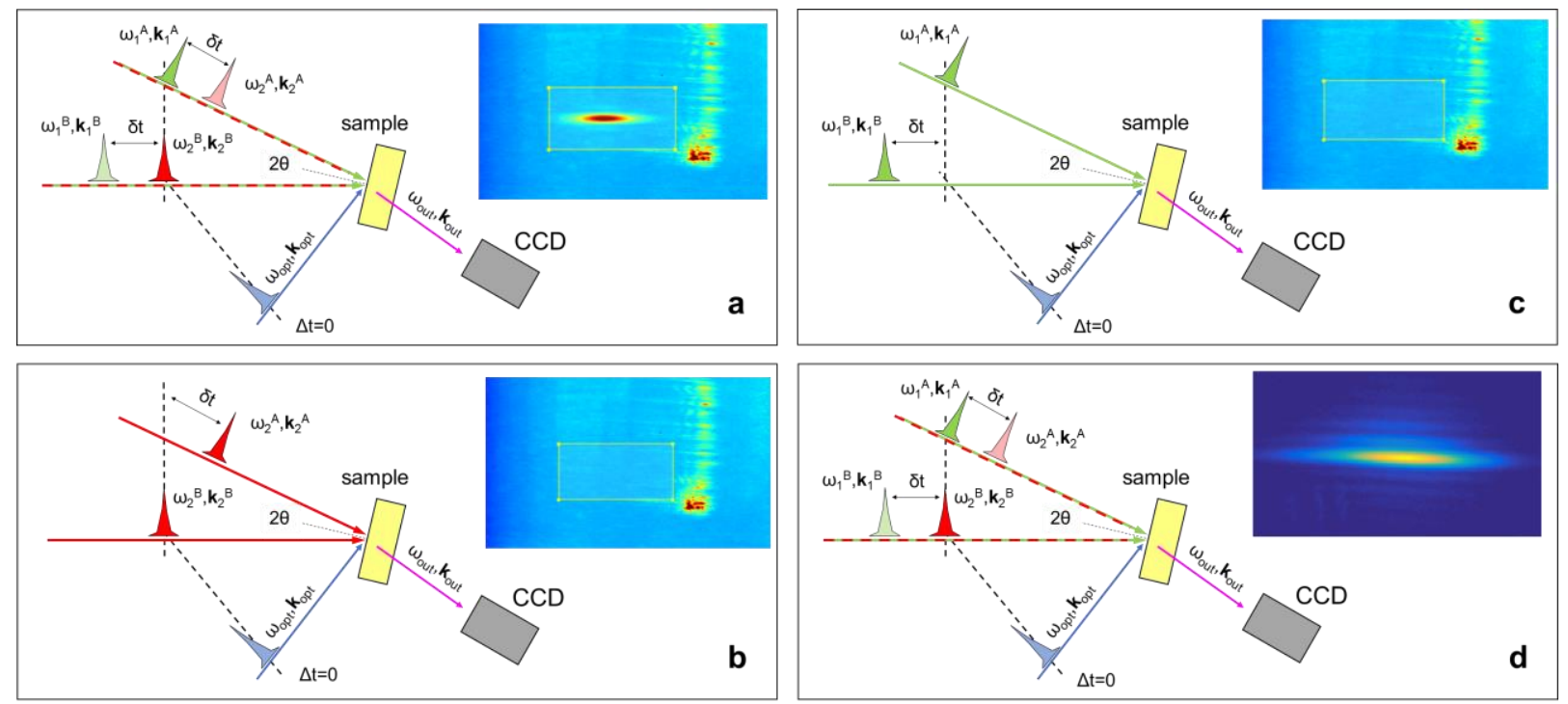

Figure 4: Pulse sequences and relative raw CCD images corresponding to: (a) both FEL pulses at the sample and (b and c) pulse $\omega_{1}$ or $\omega_{2}$ shuttered down, respectively. The yellow rectangle indicates the region where the FWM is observed, while the light in the righthand side of the yellow box is spurious scattering from the probing optical laser. Panel $\mathbf{d}$ (same pulse sequence as in a) shows a crop of the CCD image in the signal beam region after subtraction of a background image where only the optical laser probe was impinging onto the sample.

\section{CONCLUSIONS}

In summary, we have realized an experimental approach able to generate a detectable FWM (see also Sect. 2 [42]) response stimulated by ultrafast EUV FEL pulses at different photon frequencies, undoubtedly demonstrating the feasibility of multi-color FEL-based FWM experiments from a solid-state sample. This approach can be naturally extended to a larger range of samples, including molecules in solution or gas phase samples, as well as to shorter wavelengths and pulse durations. The exploitation of EUV and soft x-ray core resonances in the multi-color FWM process is in our plans, similar to what already done for EUV TG. ${ }^{15}$ This first successful step provided indications for improving the experiment in many aspects, in particular concerning the spectral analysis of the FWM signal beam and the control on other relevant experimental variables, as for instance the time delay between the FEL pulses. In a wider perspective, this may pave the way to a systematic exploitation of multi-color FEL pulses for implementing the FWM approach in the EUV and X-ray regime, an objective that has already led to relevant efforts on theoretical grounds $s^{5-7}$ and is remarkably stimulating the development of FEL technology..$^{37-41}$

\section{REFERENCES}

[1] Boyd, W. R., Nonlinear optics, Academic Press (2008).

[2] Foster, M. A., Turner, A. C., Sharping, J. E., Schmidt, B. S., Lipson, M. and Gaeta, A. L., "Broad-band optical parametric gain on a silicon photonic chip", Nature 441, 960-963 (2006).

[3] Chen, P.-Y. and Al, A., "Subwavelength imaging using phase-conjugating nonlinear nanoantenna arrays", Nano Letters 11, 5514-5518 (2011).

[4] Boyer, V., Marino, A. M., Pooser, R. C. and Lett, P. D., "Entangled images from four-wave mixing", Science 321, 544-547 (2008). 
[5] Tanaka, S. and Mukamel, S., "Coherent X-Ray Raman Spectroscopy: A Nonlinear Local Probe for Electronic Excitations", Physical Review Letters 89, 043001 (2002).

[6] Kowalewski, M., Fingerhut, B. P., Dorfman, K. E., Bennett, K. and Mukamel, S., "Simulating Coherent Multidimensional Spectroscopy of Nonadiabatic Molecular Processes: From the Infrared to the X-ray Regime", Chemical Review 117, 12165-12226 (2017).

[7] Kowalewski, M., Bennett, K., Dorfman, K. E. and Mukamel, S., "Catching Conical Intersections in the Act: Monitoring Transient Electronic Coherences by Attosecond Stimulated X-Ray Raman Signals", Physical Review Letters 115, 193003 (2015).

[8] Seddon, E. A., Clarke, J. A., Dunning, D. J., Masciovecchio, C., Milne, C. J., Parmigiani, F., Rugg, D., Spence, J. C. H., Thompson, N. R., Ueda, K., Vinko, S. M., Wark, J. S. and Wurth, W., "Short-wavelength free-electron laser sources and science: a review", Reports on Progress in Physics 80, 115901 (2017).

[9] Glover, T. E. et al., "X-ray and optical wave mixing", Nature 488, 603-608 (2012).

[10] Shwartz, S. et al., "X-Ray Second Harmonic Generation", Physical Review Letters 112, 163901 (2014).

[11] Tamasaku, K. et al. "X-ray two-photon absorption competing against single and sequential multiphoton processes", Nature Physics 9, 313-316 (2014).

[12] Lam, R. K. et al., "Soft X-Ray Second Harmonic Generation as an Interfacial Probe", Physical Review Letters 120, 023901 (2018).

[13] Bencivenga, F. et al., "Four-wave-mixing experiments with seeded free electron lasers", Faraday Discussions. 194, 283-303 (2016).

[14] Bencivenga, F. et al., "Four wave mixing experiments with extreme ultraviolet transient gratings", Nature 520, 205 (2015).

[15] Bohinc, R., et al., "Nonlinear XUV-optical transient grating spectroscopy at the Si L2,3-edge", Applied Physics Letters, 114, 181101 (2019).

[16] Naumenko, D., et al., "Thermoelasticity of Nanoscale Silicon Carbide Membranes Excited by Extreme Ultraviolet Transient Gratings: Implications for Mechanical and Thermal Management", ACS Applied Nano Materials 2, 5132-5139 (2019).

[17] Maznev, A. A., et al., "Generation of coherent phonons by coherent extreme ultraviolet radiation in a transient grating experiment”, Applied Physics Letters 113, 221905 (2018).

[18] Foglia, L., et al. "Exploring the multiparameter nature of EUV-visible wave mixing at the FERMI FEL", Structural Dynamics 6, 40901 (2019).

[19] Foglia, L., et al., "First Evidence of Purely Extreme-Ultraviolet Four-Wave Mixing", Physical Review Letters 120, 263901 (2018).

[20] Bencivenga, F. et al., "Nanoscale transient gratings excited and probed by extreme ultraviolet femtosecond pulses", Science Advances 5, eaaw5805 (2019).

[21] Begley, R. F., Harveyt, A. B. and Byer, R. L., "Coherent anti-Stokes Raman spectroscopy", Applied Physics Letters 25, 387-390 (1974).

[22] Karavitis, M., Zadoyan, R., and Ara Apkarian, V., "Time resolved coherent anti-Stokes Raman scattering of I2 isolated in matrix argon: Vibrational dynamics on the ground electronic state", Journal of Chemical Physics 114,4131 (2001).

[23] Walser, A. M., Meisinger, M., Radi, P. P., Gerber, T. and Knopp, G., "Resonant UV- fs-TCFWM spectroscopy on formaldehyde", Physical Chemistry Chemical Physics 11, 8456 - 8466 (2009).

[24] Bencivenga, F. et al., "Multi-colour pulses from seeded free-electron-lasers: towards the development of nonlinear core-level coherent spectroscopies", Faraday Discussions 171, 487-503 (2014).

[25] Bencivenga, F. et al., "FEL-based transient grating spectroscopy", Proceedings of SPIE 9512, 951212 (2015).

[26] Capotondi, F., Foglia, L., Kiskinova, M., Masciovecchio, C., Mincigrucci, R., Naumenko, D., Pedersoli, E., Simoncig, A. and Bencivenga, F., "Characterization of ultrafast free electron laser pulses using extreme ultraviolet transient gratings", Journal of Synchrotron Radiation 25, $32-38$ (2018).

[27] Mincigrucci, R., et al., "Advances in instrumentation for FEL-based four-wave-mixing experiments", NIMA 907, 132-148 (2018).

[28] Allaria, E. et al., "Two-colour pump-probe experiments with a twin pulse-seed extreme ultraviolet free-electron laser", Nature Communications 4, 2476 (2013).

[29] Allaria, E. et al., "Highly coherent and stable pulses from the FERMI seeded free-electron laser in the extreme ultraviolet", Nature Photonics 6, 699-704 (2012). 
[30] Capotondi, F., Pedersoli, E., Bencivenga, F., Manfredda, M., Mahne, N., Raimondi, L., Svetina, C., Zangrando, M., Demidovich, A., Nikolov, I., Danailov, M. B., Masciovecchio, C. and Kiskinova, M., "Multipurpose endstation for coherent diffraction imaging and scattering at FERMI@Elettra free-electron laser facility", Journal of Synchrotron Radiation 22, 544-552, (2015).

[31] Penco, G., Perosa, G., Allaria, E., Di Mitri, S., Ferrari, E., Giannessi, L., Spampinati, S., Spezzani, C. and Veronese, "M., Enhanced seeded free electron laser performance with a "cold" electron beam", Phys. Rev. Accel. Beams 23, 120704 (2020).

[32] Danailov, M. B. et al., "Towards jitter-free pump-probe measurements at seeded free electron laser facilities", Optical Express 22, 12869-12879 (2014).

[33] Cinquegrana, P. et al., "Optical beam transport to a remote location for low jitter pump-probe experiments with a free electron laser", Phys. Rev. ST Accel. Beams 17, 040702 (2014).

[34] Zangrando, M., Cocco, D., Fava, C., Gerusina, S., Gobessi, R., Mahne, N., Mazzucco, E., Raimondi, L., Rumiz, L. and Svetina C., "Recent results of PADReS, the Photon Analysis Delivery and REduction System, from the FERMI FEL commissioning and user operations", Journal of Synchrotron Radiation 22, 565-570 (2015).

[35] Svetina, C., Cocco, D., Mahne, N., Raimondi, L., Ferrari, E. and Zangrando, M., "PRESTO, the on-line photon energy spectrometer at FERMI: design, features and commissioning results", Journal of Synchrotron Radiation 23, 35-42 (2016).

[36] W. K. Peters et al., "All-optical single shot complete electric field measurement of extreme ultraviolet free electron laser pulses", submitted.

[37] Marcus, G., Penn, G. and Zholents, A. A., "Free-Electron Laser Design for Four-Wave Mixing Experiments with Soft-X-Ray Pulses", Physical Review Letters 113, 024801 (2014).

[38] Lutman, A. A., Coffee, R., Ding, Y., Huang, Z., Krzywinski, J., Maxwell, T., Messerschmidt, M. and Nuhn, H.D., "Experimental Demonstration of Femtosecond Two-Color X-Ray Free-Electron Lasers", Physical Review Letters 110, 134801 (2013).

[39] Lutman, A. A. et al., "Fresh-slice multicolour X-ray free-electron lasers", Nature Photonics 10, 745-750 (2016).

[40] Ferrari, E. et al., "Widely tunable two-colour seeded free-electron laser source for resonant-pump resonantprobe magnetic scattering", Nature Communications 7, 10343 (2016).

[41] De Ninno, G., Mahieu, B., Allaria, E., Giannessi, L. and Spampinati, S., "Chirped seeded free-electron lasers: self-standing light sources for two-color pump-probe experiments", Physical Review Letters 110, 064801 (2013)

[42] D. Bleiner, The Science and Technology of X-ray Lasers: A 2020 Update Proc. SPIE 11886, 1188602 (2021) 